\title{
Método de investigación en psicoterapia sistémica y subjetividad
}

$\mathrm{E}^{1}$ método aplicado en este proyecto abarca varios tópicos, conforme a los dos principales fenómenos abordados: el proceso psicoterapéutico en sí y la transformación de las subjetividades de todos los participantes en dicho proceso. No obstante, se consideró que para dar cuenta de los fenómenos centrales de la investigación la mejor aproximación era el análisis de casos en profundidad por varias razones.

En primer lugar, como afirma Gilgun (2011), el estudio de casos es valioso por su contribución a la teoría. Un estudio de caso es una mirada intensiva a una unidad individual, que aquí está constituida por el análisis del proceso completo de cada uno de los dos casos atendidos en psicoterapia sistémica, que se presentan como ilustración de los fenómenos estudiados. Se puede considerar que estos son casos complejos en el sentido de que abarcan múltiples elementos en interacción y la investigación-intervención se enfoca en esas múltiples interacciones, con la intención de identificar cómo las subjetividades de los participantes emergen y se transforman a través de la conversación terapéutica. Esa complejidad de cada caso y el intento de exhaustividad en el análisis hacen además que sea excesiva para un texto como este, la presentación de un mayor número de casos, aunque dentro de esta investigación se abordaron seis casos más. 
Se intenta establecer esas conexiones con base en un pensamiento abductivo, el cual se mueve a partir de indicios para identificar las pautas que dan vida a los sistemas donde los consultantes despliegan su existencia y al sistema terapéutico conformado por consultantes y terapeutas. Esta combinación investigación-intervención conduce a que se mezclen también las hipótesis terapéuticas con las de investigación en el sentido de que ambas tienen la misma pretensión de evidenciar la transformación de las subjetividades. La diferencia aparece en la perspectiva de la mirada, en el sentido de que en la conversación terapéutica circulan fundamentalmente las aproximaciones en primera y segunda persona, en tanto en la investigación predomina la aproximación en tercera persona. No obstante, como se verá en el análisis de los casos, esas distinciones terminan siendo más académicas que taxativas en el curso de la investigación-intervención, entendiendo que la utilidad de esas diferenciaciones es justamente la posibilidad de dilucidar distintas facetas de las realidades construidas y con ello generar conocimientos útiles para los consultantes en primer lugar y para los terapeutas e investigadores actores del proceso y responsables de organizar estas versiones de lo vivido de manera que se conviertan en insumos para quienes se interesan en estos temas.

Como se verá en el análisis de los casos, uno de los ingredientes de la investigación-intervención es justamente la exigencia de ir aplicando conceptos y formulando hipótesis durante el proceso para las situaciones específicas que se suscitan en la acción, pero que son explicadas y amplificadas tanto en el curso de la psicoterapia, como después, cuando el texto de esa conversación se convierte en material de investigación. No obstante, la validez de las hipótesis se va confirmando durante el proceso terapéutico en la medida en que resultan relevantes y pertinentes para los consultantes.

No sobra recordar que, según Charles Peirce, la abducción es el único tipo de argumento con el que surge una nueva idea. Porque en su concepto, ni la inducción ni la deducción pueden aportar la más mínima información a los datos de la percepción y las meras percepciones no constituyen ningún conocimiento aplicable a ningún uso práctico o teórico. Por lo tanto el conocimiento se hace por vía de la abducción. 
En este sentido, la abducción se apoya en la percepción inconsciente de conexiones entre aspectos del mundo y produce cierta emoción, lo cual la diferencia de la inducción y de la deducción. En una inferencia hipotética, esta sensación compleja es reemplazada por una única sensación de mayor intensidad, que pertenece al acto de formular la conclusión hipotética cuyo resultado, es en palabras de Peirce, una única perturbación armoniosa que se llama emoción (T.A. Sebeok y J. Umiker-Sebeok, 1987).

Puede decirse que la psicoterapia no es otra cosa que un proceso constructivo de hipótesis abductivas que juegan todo el tiempo con las conjeturas en un mundo perceptible de posibilidades, en proceso de lectura y relectura continuas de los indicios y su conexión permanente. Se trata de un razonamiento que descarta y anticipa y, a la manera de la navaja de Occam, se queda con las conexiones más relevantes de los hechos, de modo que la acción se dirige y se reorienta conforme se interpreta y se actúa. De esta forma se movilizan simultáneamente creencias, mitos y epistemes a través de la interacción psicoterapéutica que acoge ciertas características del ritual.

Todo lo anterior se enmarca en una estrategia de investigación basada en la modelización sistémica tanto en la psicoterapia como en las conversaciones en tercera persona por parte de los investigadores. Como la define Hernández (2010): “la modelización sistémica expresa esa meditación del sujeto sobre el objeto que toma siempre la forma de proyecto, a la vez teleológico (¿con cuál finalidad?), proyectivo (¿para vivir qué?) y fenomenológico (¿cuáles acciones y vivencias?). Es útil recordar que la modelización sistémica difiere de la modelización analítica en que privilegia la modelización del "acto" y no de la "cosa".

Esto significa que para el diseño de la investigación se contemplaron tres grandes referentes en coherencia con la modelización sistémica y con los objetivos propuestos:

1. Sobre el modelo de psicoterapia:

Se decidió que cada uno de los dos terapeutas abordará los casos a su cargo desde el modelo de intervención que ha desarrollado en su experiencia, dado que el estudio no busca validar cada modelo ni 
compararlo con el otro, sino tomar el proceso como contexto investigativo para observar la transformación de las subjetividades, conforme al objetivo de la investigación. Por otra parte, como justamente lo que se pretende es apreciar la presencia de la subjetividad, aunque los dos terapeutas comparten el mismo marco de referencia, se asume que cada uno lo encarna de acuerdo con sus propias particularidades, tal como se evidencia en el análisis de los casos.

Sí hubo una decisión inicial sobre el número de sesiones. Se acordó que sin ignorar las características de los casos y basados en la experiencia previa, se programaran cinco sesiones por caso con una frecuencia quincenal en promedio, sin perjuicio de hacer más o menos citas según las necesidades. Se tomó esta decisión porque se considera que con este número de sesiones es posible apreciar ciertos cambios y así era más viable el desarrollo de la investigación dentro de la programación de la IPs de la Universidad Santo Tomás, donde se atendieron los casos.

2. Sobre las aproximaciones a la investigación en primera, segunda y tercera persona:

Con base en la propuesta de Varela (Varela y Shear, 2005), Hernández (2014) propone una forma potenciadora de conjugar las tres aproximaciones y ponerlas en interjuego, en forma circular y simultánea, considerando que el conocimiento no ocurre por adiciones simples sino por relaciones múltiples que dan lugar a probabilidades exponenciales de construirlo (figura 1). En el proceso terapéutico la fuente de información en primera persona son los relatos y expresiones de los consultantes sobre sus experiencias y reflexiones; la información en segunda persona surge en la conversación terapéutica y la de tercera persona es la información que emerge principalmente en el rol de los terapeutas como investigadores, desde cuya metamirada se organiza y se sistematizan los hallazgos que corresponden al reporte investigativo. 
Figura 1. Interjuego de primera, segunda y tercera persona en el estudio de la subjetividad en psicoterapia sistémica

\section{PROCESOS EN LARED INTERSUBJETIVA DE CONSTRUCCIÓN CONJUNTA DE CONOCIMIENTO}

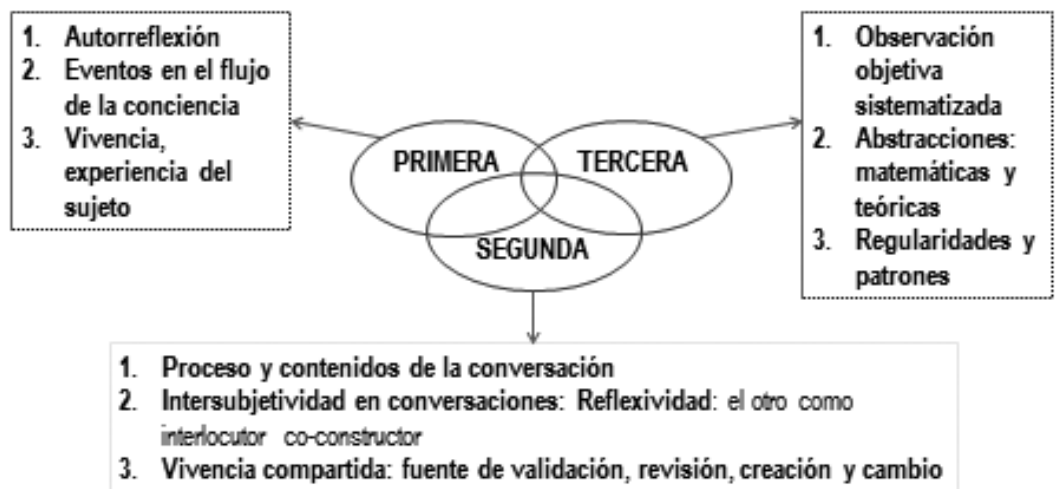

Ref: 1. Fuente de información, 2. Tpo de procesamiento de información, 3. Dimensión epistemológica

Fuente: diseñado por Hernández (2014).

Adicionalmente, los tipos de información y las formas de procesarla son diferentes en cada aproximación al fenómeno. En la primera persona son los eventos que circulan en el flujo interminable de la conciencia; en la tercera persona las abstracciones teóricas y en la segunda, las construcciones de sentido y de interacción que circulan en la conversación reflexiva terapéutica. Si se entrelazan esas tres aproximaciones, se generan productos de conocimiento válidos y específicos en cada espacio de articulación (figura 2). Así, entre la primera y la segunda persona hay un proceso de construcción, validación y cambio de las experiencias de los sujetos participantes en el sistema terapéutico. 
Figura 2. Productos de cada interfase en el interjuego primera, segunda y tercera persona

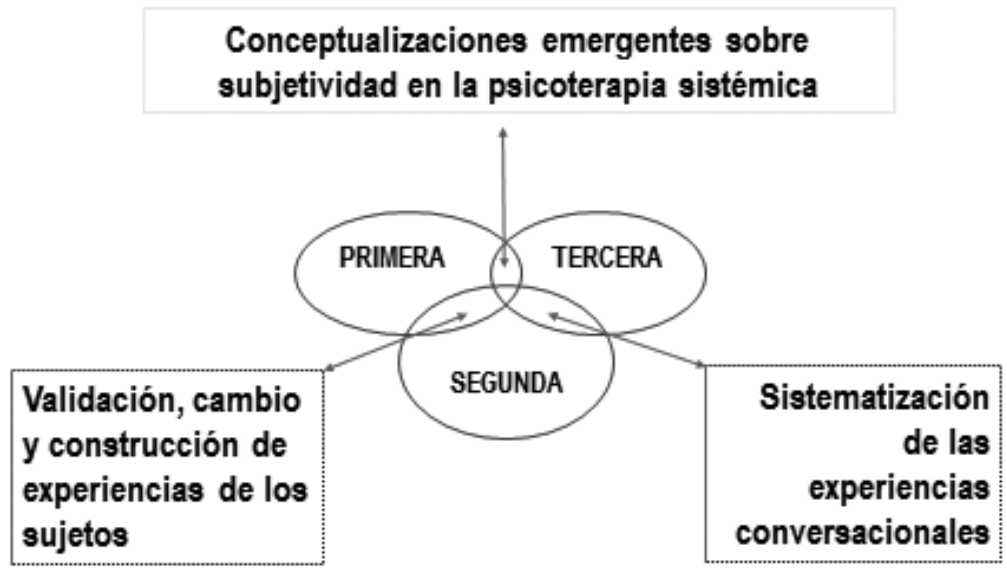

Fuente: diseñado por Hernández (2014).

Entre la segunda y la tercera persona se tendría como resultado la sistematización de las experiencias conversacionales, lo cual en términos de esta investigación equivale a la sistematización de las etapas del proceso y de los fenómenos propios de la subjetividad en psicoterapia. Entre la primera y la tercera persona resultarían las regularidades emergentes de contrastar múltiples experiencias individuales que constituyen conceptualizaciones y comprensiones sobre los temas abordados en la investigación.

1. Sobre la metodología para el análisis de los casos:

Los insumos para el análisis de los casos con fines investigativos fueron las transcripciones de todas las sesiones realizadas. Sobre ellas se hizo el siguiente proceso:

-Separación del texto de la conversación en escenas, tomando la definición de escena en teatro. Se considera que una escena es uno de los distintos fragmentos, partes, o núcleos unitarios de acción que componen un acto. La escena es un conjunto de acciones subsecuentes que conforman una unidad de acción-situación, 
temática o argumental, que sucede en un mismo espacio y tiempo, y sirve para explicar, modificar o desarrollar algún aspecto de la evolución de los personajes y de las tramas.

- Análisis del texto basado en las siguientes distinciones:

- Referentes en resonancia simultánea (figura 3).

- Fases del proceso terapéutico en términos de decisiones y puntos de giro.

- Indicadores del cambio en el self narrativo a través de proceso.

Figura 3. Referentes en resonancia simultánea

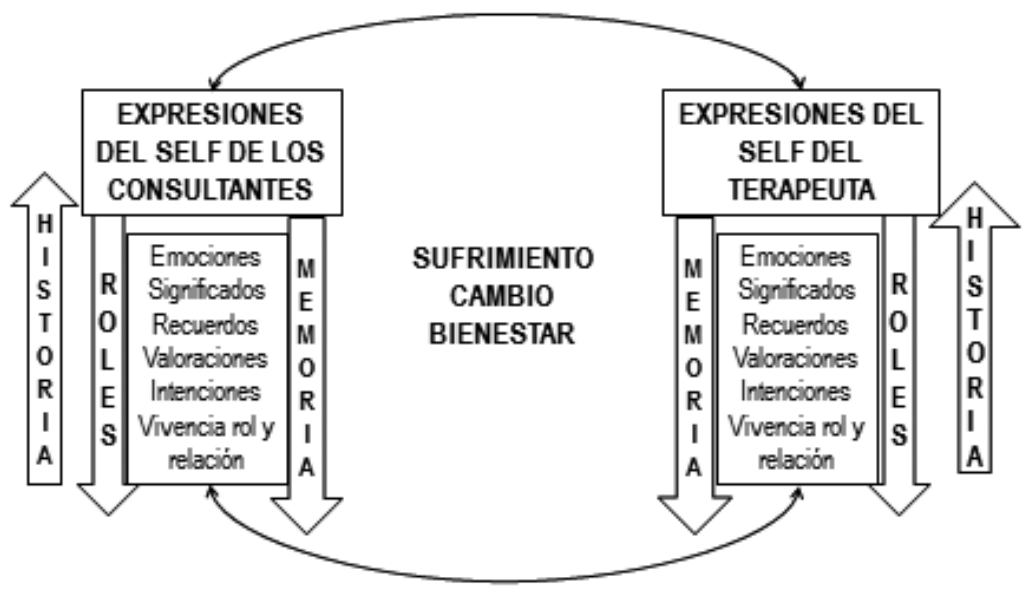

Fuente: diseño de los autores.

- Conversaciones reflexivas entre los terapeutas-investigadores para revelar los procesos auto y hetero referenciales en el curso de la terapia y en función de la investigación. 
